\title{
THE NEURAL CORRELATES OF IMPLICIT THEORY VIOLATION
}

\author{
by
}

Xiaowen $\mathrm{Xu}$

A thesis submitted in conformity with the requirements

for the degree of Masters of Arts

Department of Psychology

University of Toronto

C) Copyright by Xiaowen Xu 2011 


\title{
The Neural Correlates of Implicit Theory Violation
}

\author{
Xiaowen $\mathrm{Xu}$ \\ Masters of Arts \\ Department of Psychology \\ University of Toronto
}

2011

\begin{abstract}
Implicit theories play an important role in the structure and maintenance of people's sense of meaning, and violations to one's theory can create significant distress. Using electroencephalography (EEG), this study examined the neural correlates of implicit theory violation. Participants were primed with one of two implicit theories of success attainment and were then shown a series of words that either confirmed or violated that theory. Analyses revealed that compared to implicit theory confirmations, implicit theory violations produced greater N400 amplitudes, which is a brain wave associated with "semantic violations," or violations of meaning. Current literature on the $\mathrm{N} 400$ has been limited to language-specific semantic violations. Therefore, these results represent a novel extension of the N400 to include violations of complex lay theories of human behavior. Furthermore, these data suggest that implicit theories represent a fundamental type of semantic knowledge that helps perceivers to generate predictions about the social world.
\end{abstract}




\section{Acknowledgments}

I would like to thank my advisor, Jason Plaks, for all his help and encouragement throughout the past year. I would like to thank my other advisor, Jordan Peterson, for all of his support and guidance. I am both grateful and lucky to have two such great graduate advisors. I would also like to thank my third committee member, Michael Inzlicht, for his continuous enthusiasm and insightful suggestions regarding this study.

I am very thankful to my wonderful lab mates and friends in the program for all their moral support and advice.

I must also thank my team of hardworking research assistants, who helped me tremendously in the study's data acquisition.

Of course, I need to thank my parents for all their love and support, especially their support with my choice of going to graduate school in psychology. Last but not least, I am truly grateful towards my boyfriend for all his love, care, support, and patience for me over the years. 


\section{Table of Contents}

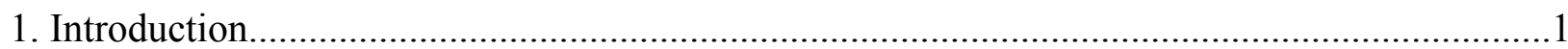

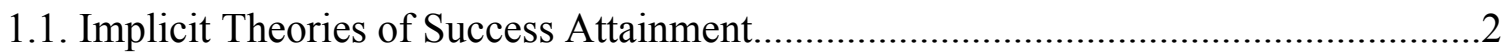

1.2. Event-Related Potentials and Implicit Theories................................. 4

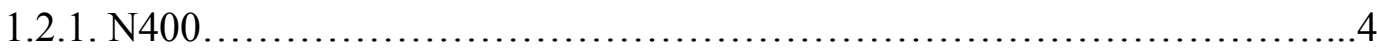

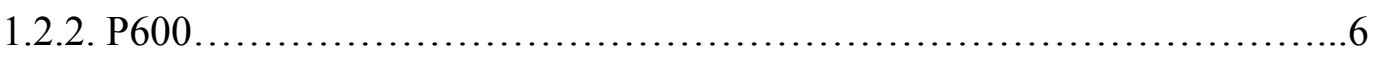

1.3. Event-Related Potentials in Social Cognition........................................

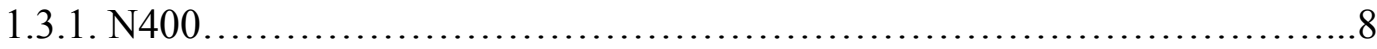

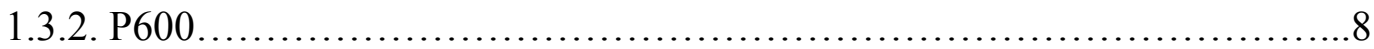

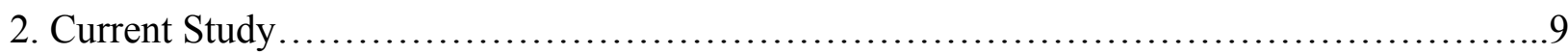

2.1. Hypotheses.................................................................. 10

2.2. Methods.........................................................................

2.2.1. Participants....................................................... 11

2.2.2. Materials.......................................................... 11

2.2.2.1. Priming Exercise.............................................11

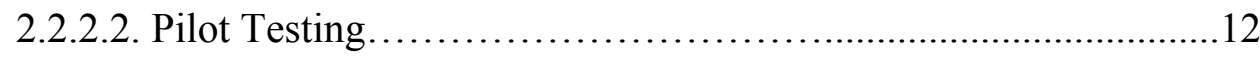

2.2.2.3. Computer Task............................................12

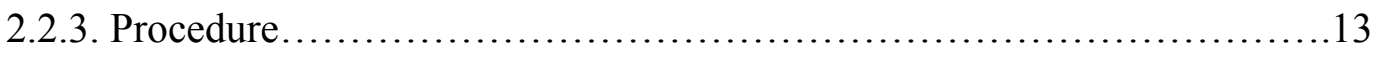

2.2.3.1. Electrophysiological Recording and Processing..................13

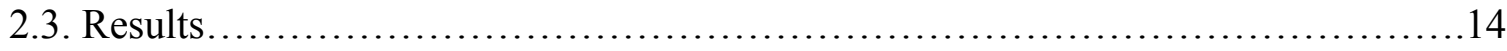


2.3.1. Accuracy and Reaction Time.

2.3.2. N400 Amplitudes.................................................15

2.3.3. P600 Amplitudes................................................... 15

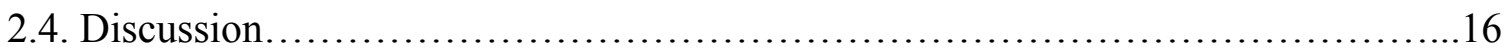

2.4.1. Future Directions ............................................... 18

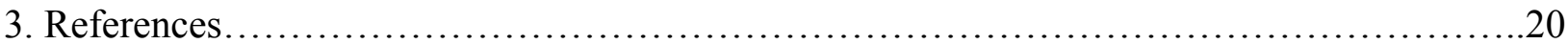




\section{Tables}

Table 1. Accuracy rate and reaction time for different word types........................23

Table 2. N400 and P600 amplitudes for different word types............................24 


\section{Figures}

Figure 1. N400 amplitudes (at Pz) for different word types.............................25 


\section{Appendices}

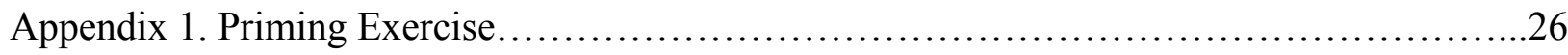




\section{Introduction}

Every day we are faced with a multitude of human behaviours that, in theory, should be perceived as highly complex and difficult to understand. Most of the time, however, we are able to decode and explain human behaviours quite precisely and effortlessly. Just how do we manage to accomplish this feat? Social, developmental, and cognitive psychologists have long hypothesized that people call on underlying implicit theories (also known as folk beliefs or lay theories) (Plaks, Levy, \& Dweck, 2009). Implicit theories are fundamental beliefs and assumptions about human behavior that provide explanatory frameworks for navigating through the social world. For instance, people have different theories about the development of intelligence, with some believing that intelligent remains fixed throughout life, whereas others believe that intelligence is malleable (Dweck, Chiu, \& Hong, 1995). Likewise, people differ in their theories about the attainment of success - some think that success is the result of personal hard work, and others believe that success is due to good fortune (Bryan, Dweck, Ross, Kay, \& Mislavsky, 2009).

Implicit theories play an important role in structuring and maintaining people's sense of meaning (Burton \& Plaks, in press; Plaks et al., 2009). Implicit theories are distinct from other sense-making cognitive structures, such as attitudes, values, or stereotypes, in two principal ways. First, whereas attitudes are by definition prescriptive, implicit theories are descriptive (Plaks et al., 2009). In other words, whereas attitudes and values are beliefs about what the world should be like, implicit theories are assumptions about what the world is truly like. Second, implicit theories differ from schemas and stereotypes, in that while schemas and stereotypes tend to apply to specific social situations or groups of people (e.g., people of 
different races/ethnicities), implicit theories refer to more general beliefs about human behaviour and the world.

Because of the important role implicit theories play in structuring and lending meaning to social information, people are motivated to believe that their theories are accurate. Therefore, encountering information that violates one's implicit theories can create significant distress and psychological conflict (Plaks, Grant, \& Dweck, 2005; Plaks \& Stecher, 2007). For example, research has shown that people exhibit higher levels of anxiety after being exposed to information that violates their core implicit theory (Plaks \& Stecher, 2007). In one study, participants who believed that intelligence remains fixed throughout life were told that their performance on an intelligence test had either declined or even improved over a period of training. Results revealed that these participants reported greater levels of anxiety upon this feedback, even when the feedback was positive. These participants, however, did not report higher levels of anxiety to feedback indicating that their performance remained unchanged following training. This demonstrates that implicit theories are of such importance to the understanding of human behaviour that people would rather receive neutral information that confirms their theory than positive information that violates their theory.

\subsection{Implicit Theories of Success Attainment}

How people come to attain success and achieve their goals is a topic of great interest to psychologists and non-psychologists alike. Recent research indicates that people have different implicit theories about how success is attained (Bryan et al., 2009). One prevalent theory holds that success is mainly the product of good fortune, i.e., receiving social opportunities and help from others. The counterpart theory states that success is primarily the 
result of personal merit, such as hard work, self-discipline, and wise decision-making. Although people may generally hold one theory more strongly than the other, it is possible in a laboratory setting to prime people with either theory, given that each theory has intuitive appeal (Bryan et al., 2009).

The good fortune and personal merit implicit theories have been found to predict differences in political orientation (Bryan et al. 2009). Indeed even the temporary activation of either theory can result in changes in people's political preferences. For example, in a study by Bryan and colleagues (2009), participants were primed with either the good fortune or personal merit theory through writing about how they gained admission into a top university. In the good fortune condition, participants were instructed to focus on the role that social opportunities and help from others played in them getting into the university, while in the personal merit condition, participants were asked to emphasize the role of personal hard word and wise decision-making. After participants were primed with either theory, they were then asked to complete questionnaires on decision-making related to political policies, e.g. whether or not the government should spend more money to build more prisons, or whether a single mother should continue to receive welfare. The researchers found that participants primed with the good fortune theory indicated support for more liberal policies than participants primed with the personal merit theory, regardless of their prior political preference (Bryan et al., 2009). In the present study, we examined neurophysiological processes associated with the experience of having one's activated theory confirmed or violated. 


\subsection{Event-Related Potentials and Implicit Theories}

Despite the advances in research on implicit theories, numerous questions about the cognitive and affective processes invoked by the psychological experience of theory violation remain unanswered. What particular cognitive processes are activated when people try to reconcile the new inconsistent information with their prior beliefs? Given that certain neural responses have been linked to specific psychological experiences, neurophysiological measures represent a potent new tool for answering such questions. The literature on one such measure, event-related potentials (ERP), has linked particular components of neural activation with specific social-cognitive processes.

\subsubsection{N400}

One potentially relevant component is the "N400" - a negative-going wave peaking approximately 400ms after stimulus onset (Kutas \& Hillyard, 1980). Cognitive neuroscientists have studied the N400 extensively, with the predominant finding that the N400 is involved in the processing of information related to semantics and violations of meaning. Numerous studies have provided consistent evidence that N400 amplitudes are elicited when people encounter semantic inconsistencies (Kutas \& Federmeier, 2000; Kutas \& Hillyard, 1980; Debruille, 2007). For instance, in one influential early study, a semantically inconsistent sentence such as "He took a sip from the transmitter" elicited a greater N400 component than a semantically meaningful sentence such as "He took a sip from the cup" (Kutas \& Hillyard, 1980). As well, semantically or associatively related stimuli (e.g., sugar or bee) presented prior to a target (e.g., honey) can serve as primes that reduce the N400 response to the target (Kutas \& Federmeier, 2000). Furthermore, it has been 
shown that the amplitude of the N400 varies with the degree of expected semantic consistency. For example, a sentence that is strongly semantically inconsistent (i.e., "Eleanor drank coffee out of a knife") elicits a larger N400 component than a sentence that is only moderately semantically inconsistent (i.e., "Eleanor drank coffee out of a bowl;" Kutas \& Federmeier, 2000). As well, the difference in N400 amplitudes between a semantically expected versus unexpected stimulus is smaller if the link between the two are stronger. For example, although "football" is the expected answer to the sentence "He scored a touchdown at the game of ...," a smaller N400 amplitude is produced when participants are shown the word "baseball" than if they were shown the word "monopoly," due to the fact that football bears much more similarities to baseball than to monopoly (Kutas \& Federmeier, 2000).

More recent work suggests that the N400 is not only involved in the processing of verbal semantics (i.e., words and sentences), but also plays a role in the processing of visual semantics such as pictures. In a study by Mudrik, Lamy, and Deouell (2010), participants were shown images that were either congruent or incongruent to visual contexts (e.g., a man playing a violin versus a man playing a broomstick, or a man dribbling a basketball versus a man dribbling a watermelon). It was found that as with verbal semantic violations, the incongruent visual contexts also elicited an N400 response. The results confirm that semantic violations affect the processing of visual contexts from an early stage (Mudrik et al., 2010). As well, the N400 appears to be involved in more than just the processing of language and verbal semantics, but may extend to the processing of broader categories semantic violations.

Thus far, we have seen that the N400 component is consistently elicited when people are faced with information that violates semantics and meaning. The prevailing view in cognitive neuroscience holds that the N400 is thus an index of people's efforts to integrate 
new, inconsistent information into their existing semantic contexts held in the long-term memory (Kutas \& Federmeier, 2000; Debruille, 2007). This integration occurs early, and the N400 amplitude is proportional to the effort required to integrate information into semantic context. Thus, stimuli that are more expected and coherent, and stimuli that fit better with the given context, are assessed and processed more easily (Kutas \& Federmeier, 2000; Debruille, 2007).

\subsection{2. $\mathrm{P} 600$}

Another relevant component is the "P600," which is a positive-going waveform peaking approximately 500-600ms post-stimulus (Kim \& Osterhout, 2005; Osterhout \& Holcomb, 1992). The majority of research on the P600 has been in the area of language processing. In contrast to the N400, which is related to meaning and semantics, findings indicate that the $\mathrm{P} 600$ is primarily involved in the processing of syntactic and structural information within a sentence. This waveform is elicited by violations involving phrase structure, agreement, verb subcategorization, and constituent-movement, and remains robust across different types of languages and various methodological factors (Kim \& Osterhout, 2005). A greater P600 waveform is usually elicited by stimuli that are inconsistent with the expected structure or syntax of a sentence, e.g. a grammatically incorrect sentence such as "John hoped the man to leave" (Osterhout \& Holcomb, 1992).

More recent research revealed that the P600 effect does not only result from syntactic inconsistencies in sentences, but can even be elicited by incongruent "syntax" in arithmetical word problems (Fisher, Bassok, \& Osterhout, 2010). When people reason about arithmetic

problems, they tend to analogically align categorically related objects (e.g., roses and daisies) 
with addition and functionally related objects (e.g., roses and vases) with division. In the study, participants were shown a series of arithmetic word problems that were either analogically aligned or misaligned, and were either arithmetically correct or incorrect. It was shown that incorrect arithmetic (e.g., twelve roses plus three daisies equals to sixteen) elicited an N400 response, whereas misaligned pairings of arithmetic objects (e.g., twelve roses plus three vases equals to fifteen) elicited a P600 effect, regardless of the correctness of the actual arithmetic (Fisher et al., 2010). These results indicate that the P600 can be used as an index of analogical incongruities.

\subsection{Event-Related Potentials in Social Cognition}

Although there exists a plethora of research looking at ERP components and their roles in the processing of basic semantic or syntactic violations, such as the perception of sentences or images, little work has been done to investigate how these components may be related to the processing of more complex social phenomena. Given what is known about the N400 and P600, it is reasonable to believe that these components could be involved in the brain's responses to more complex social behaviours. After all, it has been repeatedly demonstrated that these components are elicited when encountering inconsistent or unexpected stimuli (e.g., when reading about somebody drinking coffee from a "transmitter" as opposed to a "cup"). A social psychological interpretation of these findings may be that in these sentences, what is really violated is the expectancy that comes from the assumptions people have about the world - for example, the assumption that people tend to drink coffee from a cup and not from a transmitter. These types of expectancy or assumption violations can occur at a local level involving basic perception of sentence structures, or they may be 
concerned with a deeper level of processing, i.e. violations of social situations or behavioural norms.

\subsubsection{N400}

More recent research has begun to investigate the possibility of the involvement of the N400 in deeper and more complex social phenomena. In one study, researchers examined how the violation of social stereotypes is related to N400 activation (White, Crites Jr., Taylor, \& Corral, 2009). In this study, participants were shown word pairs depicting various gender stereotypes, with some word pairs being stereotype consistent (e.g., "women" and “caring") and others word pairs being stereotype violating (e.g., "women" and "aggressive"). The results indicated that word pairs that violated gender stereotypes led to a more pronounced N400 component, whereas the pairing of expected stereotype words resulted in a reduced N400, suggesting that it is easier for people to access and integrate stereotypeconsistent information than stereotype-violating information (White et al., 2009). This result is promising, as it demonstrates that the N400 may indeed be relevant to the processing and integration of social expectancies.

\subsubsection{P600}

In addition to the N400, it has been shown that the P600 can also be elicited by violations of social stereotypes, and not just syntactic violations. In one study, participants were asked to read sentences about various characters and their actions, and were asked to indicate whether each sentence is acceptable both semantically and syntactically (Osterhout, Bersick, \& McLaughlin, 1997). Each sentence character's gender was either definitionally 
(e.g., the bachelor) or stereotypically (e.g., the doctor) male or female. Violation of gender stereotype was achieved through the manipulation of the gendered pronoun describing each character, such as "The bachelor cooked himself/herself a meal." For both definitional and stereotypical gender types, pronouns that violated gender expectancies were shown to elicit a more pronounced P600 component than pronouns confirming gender expectancies. This effect persisted even when participants judged the sentences as acceptable and meaningful (Osterhout et al., 1997). These results appear to indicate that ERPs can reveal sensitivity to gender stereotypes that may not be detected by other types of explicit measures (for further evidence of this effect, see Bartholow, Fabiani, Gratton, \& Battencourt, 2001.)

\section{Current Study}

To the extent that the experience of theory violation shares similar neurocognitive processes with stereotype violation, we hypothesized that stimuli that violate people's implicit theories will elicit similar activation patterns (in the form of an N400 and/or P600 component) as the brain attempts to make sense of this new information. If so, this would indicate that people do experience difficulty processing information that violates their core implicit theories.

In the current study, we investigated the neural correlates of response to violations of the good fortune and personal merit implicit theories. Participants were first primed with either the good fortune or personal merit theory by reading an article describing how Warren Buffet, a renowned investor, has achieved success through good fortune or personal merit. Afterward, the participants were shown a series of stimulus words on the computer and were asked to determine whether each word (e.g., lucky) applies to the target person (e.g., Warren 
Buffett). These words either confirmed or violated the participants' previously activated theory. Participants' response times and brain activities during the task were recorded. Thus, this task assessed participants' behavioural and brain responses to traits that confirm or violate their previously activated theory.

It is important to point out that in the present paradigm, we violated the participants' implicit theory in two ways: through direct theory violation and through simple semantic violation. Direct theory violation entailed exposing the participant to traits that were opposite in meaning to traits related to their implicit theory, e.g., a word that directly contradicted the good fortune theory would be "unfortunate." A semantic violation, on the other hand, entailed traits that were completely irrelevant to either of the two primed theories (e.g., "musical" or "purple").

\subsection{Hypotheses}

Because this study was exploratory in nature and was one of the first studies to examine neural components in a social context, we developed two sets of plausible hypotheses. In the first set of hypotheses, we predicted that words that were direct theory violations (e.g., "unlucky" shown to a good fortune theorist) would elicit larger N400 amplitudes than words that that were theory confirmations (e.g., "lucky"), but would elicit smaller N400 amplitudes than words that were semantic violations (e.g., "orange"). This is because direct theory violations are more subtle than semantic violations, and involve the processing of more abstract concepts (such as traits and goals) rather than visible aspects, e.g. physical features. In the second set of hypotheses, we predicted that direct theory violations would elicit larger P600 amplitudes than words that confirm one's theory, but would elicit smaller P600 
amplitudes than words that were semantic violations. Larger N400 amplitudes would indicate a greater effort by the brain to integrate these violations into its existing semantic memory (Kutas \& Federmeier, 2000), whereas larger P600 amplitudes would reflect a context updating process, in which the current model of the target is updated in the working memory (Bartholow et al., 2001). In both cases, however, we predict that words which are direct theory violations would take a longer time to process than both theory confirming words and semantic violations.

\subsection{Method}

\subsubsection{Participants}

Participants for this study consisted of 27 first-year psychology undergraduate students (16 males) at the University of Toronto, who participated in this study for course credits. The mean age of the participants was 19.63 years $(\mathrm{SD}=1.52$ years).

\subsubsection{Materials}

\subsubsection{Priming Exercise}

To prime participants with either the good fortune or personal merit implicit theory, they were asked to read a short biography about Warren Buffett focusing on how Buffett has attained his success and achievements. In the good fortune condition, the biography was written such that Buffett's success was attributed mainly to his good fortunes and social opportunities. The biography emphasized Buffett's social advantages and the valuable help he received from others. The biography for the personal merit condition, however, focused 
on how Buffett has achieved success through his personal hard work, perseverance, and wise decision-making. It primarily emphasized Buffett's personal merits and discipline as his key to success. The complete text of the priming exercise is provided in the Appendix.

\subsubsection{Pilot Testing}

A list of words related to the good fortune and personal merit implicit theories was generated by the experimenters, with the words being either theory-confirming or theoryviolating. These words were pilot-tested for their relevance to each theory and for their familiarity. The relevance of each word was measured on a 7-point Likert scale, with 1 being "Very irrelevant" and 7 being "Very relevant." Familiarity was also measured on a 7-point scale, with 1 corresponding to "Very unfamiliar" and 7 to "Very familiar." A stimulus word was included in the experimental session only if it had a mean relevance rating of at least 3.5 and a mean familiarity rating of at least 4.5 based on the pilot test results. As a result, a total of 34 words were used for the good fortune theory, with 17 words being theory-confirming (e.g., lucky, fortunate) and 17 words being theory-violating (e.g., misfortune, tragedy). Thirty-five words were generated for the personal merit theory, where 18 words were theoryconfirming (e.g., hardworking, industrious) and the rest were theory-violating (e.g., lazy, procrastinate). An additional 25 words that are semantic anomalies (i.e. were irrelevant to either theory) were also generated (e.g., triangular, funny).

\subsubsection{Computer Task}

In the computer task, participants were asked to indicate via keyboard responses whether stimulus words presented on the computer screen applied to Warren Buffett. 
Participants were exposed to three categories of stimulus words: theory confirming, theory violating, and semantics violating. Each stimulus word was repeated, such that the total trial count was around 152-155 per participant. The stimulus words were presented in a pseudorandom order, such that words from the same category were not presented more than twice in a row.

\subsubsection{Procedure}

Upon arrival at the lab, participants were asked to complete a consent form. Participants were then primed with either the good fortune or personal merit implicit theory (the order randomized). Afterward, they completed the computer task, a demographics questionnaire, and were lastly debriefed and given their credits.

During the computer task, each trial consisted of the following: a fixation cross appeared on the screen for $500 \mathrm{~ms}$, followed by a blank screen of $150 \mathrm{~ms}$. The purpose of the blank screen was to serve as a buffer to ensure that neural responses to the disappearance of a stimulus (i.e., the fixation cross) would not affect processing of subsequent stimuli. The cue word "Warren Buffett" then appeared onscreen for $500 \mathrm{~ms}$, followed by the $150 \mathrm{~ms}$ blank screen. The stimulus word appeared next for $1000 \mathrm{~ms}$. The participant had $1500 \mathrm{~ms}$ to make a response to the stimulus word. After the participants gave their response, a final blank screen of 200ms appeared before the beginning of the next trial. Participants were given a break after every 20 or so trials to minimize potential effects of fatigue or habituation.

\subsubsection{Electrophysiological Recording and Processing}

Continuous EEG from 32 scalp sites was recorded during the computer task using the 
NeuroScan NuAmps amplifier system. An electrode cap with Ag/Ag/Cl-sintered electrodes was placed on the participants' heads. Horizontal and vertical eye movements were monitored using four electrodes, two placed to the side of each eye, and two placed above and below the left eye. EEG recordings were digitized at $1000 \mathrm{~Hz}$ with average-ear references and a forehead ground. Frequencies were filtered using a 1-30Hz bandpass filter $(96 \mathrm{~dB}$, zerophase shift). Movement artifacts were detected and removed using a $-75 \mathrm{mV}$ and $+75 \mathrm{mV}$ threshold. Signals were baseline corrected by subtracting the mean voltage occurring $200 \mathrm{~ms}$ pre-stimulus. Correct trials were averaged with an epoch of $200 \mathrm{~ms}$ pre-stimulus to $800 \mathrm{~ms}$ post-stimulus. The N400 was quantified as the peak minimum between 300 and $500 \mathrm{~ms}$ poststimulus at the parietal midline electrode site (Pz), and the $\mathrm{P} 600$ was quantified as the peak maximum occurring between 500 and $800 \mathrm{~ms}$ post-stimulus at the central midline electrode site $(\mathrm{Cz})$. Past research has shown that the N400 and P600 are typically most predominant at these sites (e.g., Kiang, Prugh, \& Kutas, 2010).

\subsection{Results}

Table 1 lists the accuracy rates and reaction times (correct trials only) for each category of stimuli words (theory confirming, theory violating, and semantics violating). Table 2 displays the means and SDs for the N400 and P600 amplitudes for each word category.

\subsubsection{Accuracy and Reaction Time}

Overall, participants performed well on the computer task, i.e. participants correctly identified words as applying or not applying to Warren Buffet at a rate of $78 \%$ or higher. A one-way repeated measure ANOVA indicated that there was a significant difference in 
accuracy rate between types of words (theory confirming, theory violating, semantic violating $)(F(2,52)=3.51, p<0.05)$. Participants performed better on theory violating trials than on theory confirming $(\mathrm{t}(26)=-3.37, \mathrm{p}<0.01)$ and semantics violating trials $(\mathrm{t}(26)=$ $2.52, \mathrm{p}<0.05$ ). To examine differences in reaction time between each category (see Table 2), a repeated measure ANOVA was performed on the correct trials for each category, which revealed no significant difference in reaction time $(F(2,52)=0.52, \mathrm{p}=0.60)$.

\subsubsection{N400 Amplitudes}

ERP analyses were conducted using only correct trials, as this would avoid the influence of random noise incorrect trials may contain (i.e., due to lack of attention). A repeated measure ANOVA indicated that there was a significant difference in N400 amplitudes between the three categories $(\mathrm{F}(2,52)=7.68, \mathrm{p}<0.01)$ (Figure 1). Overall, N400 amplitudes were more negative for theory violating words $(\mathrm{M}=-3.04 \mu \mathrm{V})$ than for theory confirming words $(\mathrm{M}=-2.07 \mu \mathrm{V}, \mathrm{t}(26)=2.13, \mathrm{p}<0.05, \mathrm{~d}=0.36)$. Additionally, semantics violating words $(\mathrm{M}=-4.17 \mu \mathrm{V})$ also elicited more negative $\mathrm{N} 400$ amplitudes than theory confirming words $(\mathrm{t}(26)=3.60, \mathrm{p}<0.01, \mathrm{~d}=0.78)$. The difference between theory violating and semantics violating words was marginally significant $(\mathrm{t}(26)=2.01, \mathrm{p}=0.06)$.

\subsubsection{P600 Amplitudes}

A repeated measure ANOVA did not reveal an overall effect of word type on P600 amplitude, $(F(2,52)=2.06, p=0.14)$. A t-test did reveal a significant difference between theory violating $(\mathrm{M}=4.04 \mu \mathrm{V})$ and semantics violating words $(\mathrm{M}=4.98 \mu \mathrm{V}, \mathrm{t}(26)=-2.09, \mathrm{p}$ $<0.05, \mathrm{~d}=0.47$ ), with semantics violating words eliciting a more positive P600 amplitude. 


\subsection{Discussion}

To our knowledge, this study is the first to examine how the brain processes information that violates people's implicit theories of success attainment. All participants were primed with a specific implicit theory (either good fortune or personal merit). Subsequent words that violated the theory resulted in a greater N400 response than words that confirmed the theory. Specifically, compared to theory confirming information, greater N400 amplitudes were elicited in response to both information that directly violated one's primed theory, as well as information that was semantically anomalous. The presence of the N400 in response to theory violating information may suggest that the brain is effortfully trying to integrate this new, inconsistent information into its existing semantic knowledge (Kutas \& Federmeier, 2000). Furthermore, the lack of a P600 effect may also indicate that implicit theory violations bear more similarity to semantic violations than syntactic or structural violations.

An important contribution of this study is that it provides evidence that the N400 is not only limited to language-specific semantic violations, but is also elicited by broader violations of social meanings and expectancies. In other words, the N400, rather than solely reflecting language processing, may actually be an index of general meaning or expectancy violation. If so, this may represent a contribution to the field of cognitive neuroscience, as most work in the field so far has been restricted to examining people's responses to low-level perceptual expectancy violations. These data suggest that such processes may extend - in perhaps weaker form - to the violation of more complex theories of human behaviour.

The results of this study not only provide evidence that the brain processes expectancy violations differently than expectancy confirming information, but also appears to show that 
there are different levels of processing for different types of violations. While the N400 amplitudes for both theory violations and semantic violations differ significantly from theory confirming information, the effect remains stronger for semantic violations. Indeed, if one believes that Warren Buffett is hardworking, it makes little sense for him to also be lazy, but it makes even less sense for him to be orange. Furthermore, as seen by the trending difference between N400 amplitudes for theory violations and semantic violations, it may be that the brain also differentiates between the two as different degrees of expectancy violations.

In a social psychological context, the N400 response resulting from theory violations may suggest that people are motivated to make sense of and defend their beliefs from violation, even when these beliefs are not especially deeply held and are simply the result of an experimental prime. After all, it is reasonable to believe that for most people, whether or not Warren Buffett is hardworking or lazy has little bearing on their lives in general. Nevertheless, when people were primed with a specific theory, they displayed a prominent response to information that violated this theory. The use of implicit theories also differentiates the current study from research examining stereotype violation (e.g., White et al., 2009), as it shows that the brain exhibits sensitivity to the violation of more general beliefs people have about human behaviour, not just stereotypes of specific groups (e.g., Bartholow et al., 2001; Osterhout et al., 1997). Thus, in addition to its contribution to cognitive neuroscience, the present study helps to delineate the types of social expectancies that shape perceivers' everyday interpersonal cognition. 


\subsubsection{Future Directions}

One issue worth noting is the lack of any P600 effects in the present study, given that past research on gender stereotype violations have revealed interesting P600 effects (e.g., Osterhout et al., 1997). However, this may not be entirely surprising given the methodology of the present study, which bears more similarity in design to the study by White and colleagues (2009). It is possible that by using a single stimulus word per trial, it allows for little syntactic structure and therefore would eliminate the possibility for proper syntax violations, which would result in the P600 response. Thus, if a different methodology were adopted (e.g., using a stimulus sentence instead of a single word), it may be possible then to also demonstrate the P600 effect for implicit theory violations in addition to the N400 effects. If so, this would then show that for social violations, it is not a matter of semantics or syntax, but rather all that matters is the presence of a violation. This would also reinforce the idea that these ERP components, rather than being language-specific, actually extend to a general sense of meaning and expectancy.

Another issue for future research would be to differentiate between trait versus theory violation. Because both the priming exercise and computer task in the present study involved judgments about Warren Buffett, it is possible that what was actually violated was simply people's perceived traits of Warren Buffett, rather than their implicit theories about success attainment. Thus, future work will need to extend these findings. For instance, after being primed with the good fortune/personal merit theory, instead of deciding if a word matches the description of Warren Buffett in the computer task, participants would be asked to read various sentences about the actions and subsequent consequences of a different target person 
(e.g., "Bill"), where these sentences either confirm or violate their previously activated theory. This design would then allow us to gain more certain insights into implicit theories and their violations, and would rule out the possibility of simple trait violations.

Finally, it will be of great research interest to investigate the neural correlates for the violations of other types of implicit theories, such as people's theories about intelligence or justice in the world (e.g., Lerner, 1980). This would not only contribute further to our understanding of implicit theories and their violations, but would also shed more light onto the functions of their related neural components. These studies will have the potential to contribute to both the social psychological and cognitive neuroscience literatures. 


\section{References}

Bartholow, B. D., Fabiana, M., Gratton, G., \& Battencourt, B. A. (2001). A

psychophysiological examination of cognitive processing of and affective responses to social expectancy violations. Psychological Science, 12, 197-204.

Burton, C. M., \& Plaks, J. E. (in press). Lay theories: A cornerstone of meaning. In K. D.

Markman, T. Proulx, \& M. J. Lindberg (Eds.), The psychology of meaning. Washington, DC: American Psychological Association.

Bryan, C. J., Dweck, C. S., Ross, L., Kay, A. C., \& Mislavsky, N. O. (2009). Political mindset: Effects of schema priming on liberal-conservative political positions. Journal of Experimental Social Psychology, 45, 890-895.

Debruille, J. B. (2007). The N400 potential could index a semantic inhibition. Brain Research Reviews, 56, 472-477.

Dweck, C. S., Chiu, C., \& Hong, Y. (1995). Implicit theories and their role in judgments and reactions: A world from two perspectives. Psychological Inquiry, 6, 267-285.

Fisher, K. J., Bassok, M., \& Osterhout, L. (2010). When two plus two does not equal four: Event-related potential responses to semantically incongruous arithmetic word problems. Proceedings of the Cognitive Science Society 2010. 
Kiang, M., Prugh, J., \& Kutas, M. (2010). An event-related brain potential study of schizotypal personality and associative semantic processing. International Journal of Psychophysiology, 75, 119-126.

Kim, A., \& Osterhout, L. (2005). The independence of combinatory semantic processing: Evidence from event-related potentials. Journal of Memory and Language, 52, 205225.

Kutas, M., \& Federmeirer, K. D. (2000). Electrophysiology reveals semantic memory use in language comprehension. Trends in Cognitive Neuroscience, 4, 463-470.

Kutas, M., \& Hillyard, S. A. (1980). Reading senseless sentences: Brain potentials reflect semantic incongruity. Science, 207, 203-205.

Lerner, M. J. (1980). The belief in a just world: A fundamental delusion. New York: Plenum.

Mudrik, L., Lamy, D., \& Deouell, L. Y. (2010). ERP evidence for context congruity effects during simultaneous object-scene processing. Neuropsychologia, 48, 507-517.

Osterhout, L., Bersick, M., \& McLaughlin, J. (1997). Brain potentials reflect violations of gender stereotypes. Memory \& Cognition, 25, 273-285.

Osterhout, L., \& Holcomb, P. J. (1992). Event-related brain potentials elicited by syntactic anomaly. Journal of Memory and Language, 31, 785-806. 
Plaks, J. E., Grant, H., \& Dweck, C. S. (2005). Violations of implicit theories and the sense of prediction and control: Implications for motivated person perception. Journal of Personality and Social Psychology, 88, 245-262.

Plaks, J. E., Levy, S. R., \& Dweck, C. S. (2009). Lay theories of personality: Cornerstones of meaning in social cognition. Social and Personality Psychology Compass, 3, 1-13.

Plaks, J. E., \& Stecher, K. (2007). Unexpected improvement, decline, and stasis: A prediction confidence perspective on achievement success and failure. Journal of Personality and Social Psychology, 93, 667-684.

White, K. R., Crites Jr., S. L., Taylor, J. H., \& Corral, G. (2009). Wait, what? Assessing stereotype incongruities using the N400 ERP component. Social Cognitive and Affective Neuroscience, 4, 191-198. 


\section{Table 1}

Accuracy rate and reaction time for different word types.

\begin{tabular}{|c|c|c|c|c|c|c|}
\hline & \multicolumn{2}{|c|}{ Theory Confirming } & \multicolumn{2}{|c|}{ Theory Violating } & \multicolumn{2}{|c|}{ Semantics Violating } \\
\hline & Mean & SD & Mean & SD & Mean & SD \\
\hline $\begin{array}{l}\text { Accuracy } \\
\text { Rate }\end{array}$ & $78.07(\%)$ & $19.41(\%)$ & $87.22(\%)$ & $13.47(\%)$ & $79.11(\%)$ & $15.55(\%)$ \\
\hline $\begin{array}{l}\text { Reaction } \\
\text { Time }\end{array}$ & $778.82(\mathrm{~ms})$ & $94.76(\mathrm{~ms})$ & $788.02(\mathrm{~ms})$ & $101.37(\mathrm{~ms})$ & $793.45(\mathrm{~ms})$ & $109.36(\mathrm{~ms})$ \\
\hline
\end{tabular}


Table 2

N400 and P600 amplitudes for different word types.

\begin{tabular}{|l|c|c|c|c|c|c|}
\hline & \multicolumn{2}{|c|}{ Theory Confirming } & \multicolumn{2}{c|}{ Theory Violating } & \multicolumn{2}{c|}{ Semantics Violating } \\
\cline { 2 - 7 } & Mean $(\mu V)$ & SD $(\mu V)$ & Mean $(\mu V)$ & SD $(\mu V)$ & Mean $(\mu V)$ & SD $(\mu V)$ \\
\hline N400 & -2.07 & 2.73 & -3.04 & 2.97 & -4.17 & 3.23 \\
\hline P600 & 4.66 & 2.01 & 4.04 & 1.74 & 4.98 & 1.98 \\
\hline
\end{tabular}


Figure 1

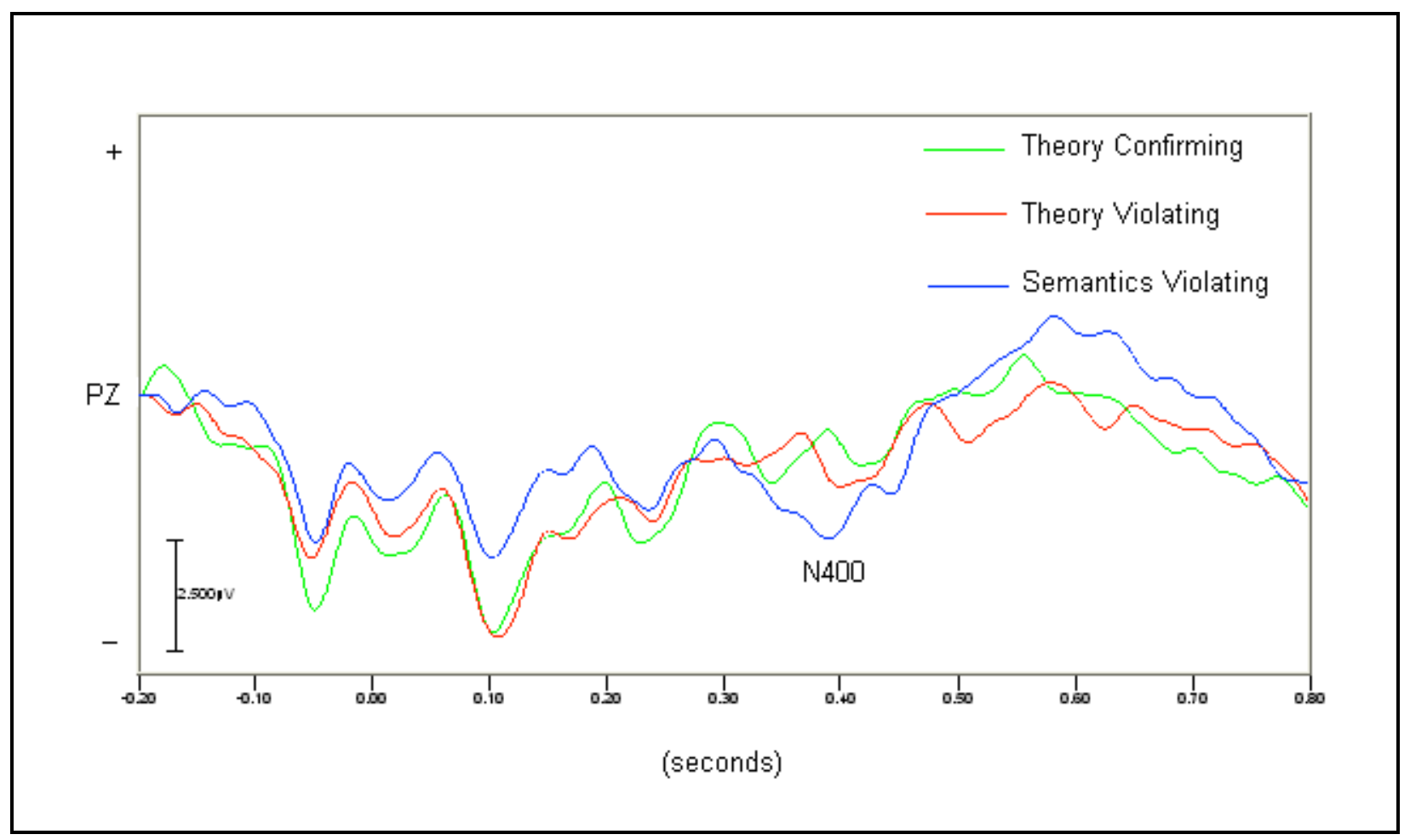

N400 amplitudes (at Pz) for different word types. 


\section{Appendix 1}

\section{Priming Exercise}

\section{Good Fortune Condition}

Warren Buffett, also known as the "Oracle of Omaha," is a successful American investor who is consistently ranked among the world's wealthiest people. Known as one of the most successful investors in the world, he is the primary shareholder, chairman, and CEO of Berkshire Hathaway. Although many factors contribute to Buffett's success, one of the key components remains in Buffett's good fortune in having certain social advantages and receiving valuable help from others around him.

Warren Buffett's father Howard was a stockbroker and later on a United States Congressman. Buffett was given the exposure to investing at an early age when he began working at his father's brokerage. Buffett attended the Columbia Business School, where he had the opportunity to study under Benjamin Graham, one of the leading investors at that time. After he graduated, Buffett worked at his father's brokerage and invested in a Texaco station and real estate, though neither of which were successful. Buffett's luck eventually changed, as he finally received an offer from Benjamin Graham to come work for him. This opportunity allowed Buffett to learn valuable insights about stock investing from Graham himself. A few years later, Buffett started his own partnership. He then purchased Berkshire Hathaway, which at first did not prove to be of much success. However, under the influence and help of his friend and business partner Charlie Munger, Buffett changed his investment approaches, which eventually led to Berkshire Hathaway becoming a giant in the investment business. 


\section{Personal Merit Condition}

Warren Buffett, also known as the "Oracle of Omaha," is a successful American investor who is consistently ranked among the world's wealthiest people. Known as one of the most successful investors in the world, he is the primary shareholder, chairman, and CEO of Berkshire Hathaway. Although many factors contribute to Buffett's success, one of the key components remains in Buffett's personal merits of working hard and consistently making wise decisions.

Warren Buffett began working at his father's brokerage at an early age and he produced many successful moneymaking ideas while he was in high school. Buffett attended the Columbia Business School, where he studied under Benjamin Graham, one of the leading investors at that time. Buffett was an excellent and hardworking student - he received the only A + Graham had ever handed out. After he graduated, Buffett worked at his father's brokerage and invested in a Texaco station and real estate, though neither of which were successful. Buffett persevered in his work and eventually received an offer from Benjamin Graham to come work for him. A few years later, Buffett started his own partnership with just a few of his relatives and friends. The partnership, which Buffett ran from his own bedroom, became very successful. He then purchased Berkshire Hathaway, which at first did not prove to be of much success. However, throughout the years, Buffett changed his investment approaches and made wise investments with the company, which eventually led to Berkshire Hathaway becoming a giant in the investment business. 